\title{
Emergence of Primary CNS Lymphoma in a Patient with Findings of CLIPPERS
}

\author{
Amy Wei Lin, Sumit Das, J. Alexander Fraser, Lee-Cyn Ang, Anita Florendo-Cumbermack, \\ Mary E. Jenkins, Keng Yeow Tay
}

Can J Neurol Sci. 2014; 41: 528-529

We report the case of a patient who had clinical, radiological, and neuropathological features initially suggestive of chronic lymphocytic inflammation with pontine perivascular enhancement responsive to steroids (CLIPPERS) but who ultimately succumbed to autopsy-proven primary central nervous system lymphoma (PCNSL). This case emphasizes the need for close follow-up of patients who are suspected to have CLIPPERS, and the importance of maintaining a continued high index of suspicion for an alternative diagnosis, particularly when such patients develop steroid-resistance.

CLIPPERS is a recently described inflammatory disease ${ }^{1-3}$ centred in the pons with common features including: (1) episodic brainstem symptoms responsive to corticosteroids and immunotherapy; (2) characteristic punctate and curvilinear gadolinium-enhancing lesions predominantly in the pons with variable involvement of other structures including the cerebellum, cerebral white matter, and spinal cord on magnetic resonance imaging (MRI); and (3) T-lymphocytic infiltrate with perivascular predominance in the brain biopsy specimens ${ }^{2}$.

The original paper on CLIPPERS, by Pittock et al in 2010, described eight patients with common clinical, radiological, and pathological features. Since that time, two other case series ${ }^{2,3}$ described an additional 17 patients with similar features. Followup periods ranged from 6 to 408 months.

It has been suggested that the combination of clinical and radiological features in CLIPPERS may be sufficiently distinctive that the disease could be diagnosed and treated without pathological examination, if alternative diagnoses are rigorously excluded ${ }^{1}$. However, making a definitive diagnosis remains challenging due to the lack of specific biomarkers and overlap in imaging features with a number of other disease entities, such as neurosarcoidosis, central nervous system (CNS) lymphoma, lymphomatoid granulomatosis, CNS vasculitis, chronic perivascular infectious process, Behçet disease, glioma, demyelinating disease, and histiocytosis ${ }^{1}$.

\section{Case Report}

Our patient was a 74-year-old male who was admitted to the inpatient Neurology service with a six month history of progressive functional decline with ataxic gait, binocular diplopia, dysarthria, and weight loss. He had no prior neurological or oncological history. On examination he also had mild right facial sided weakness. Brain and spine MRI showed multiple nodular enhancing lesions, predominantly in the pons but also involving the cerebellum, occipital white matter, and the

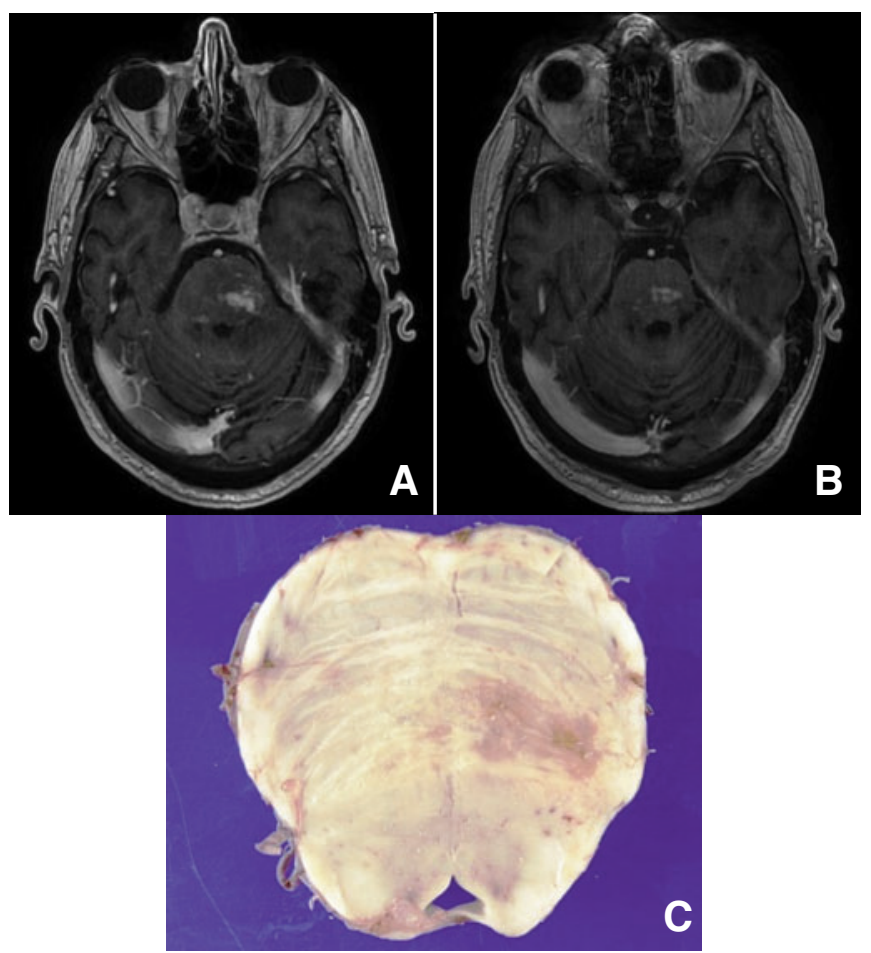

Figure 1: Representative MRI and gross pathology. (a): axial T1 gadolinium enhanced images at initial presentation showing punctate enhancing lesions predominantly in the pons. (b): repeat MRI 5 months later shows persistent pontine lesions and new enhancing lesion centred in the left internal capsule (not shown). (c): corresponding gross pathological specimen at autopsy.

spinal cord. These lesions were T2 and fluid-attenuated inversion recovery (FLAIR) hyperintense, and enhanced following gadolinium administration, without evidence for diffusion restriction (Figure 1a). Multiple high-volume lumbar punctures showed elevated protein between 0.9 to $1.1 \mathrm{~g} / \mathrm{L}$, no

From the Department of Medical Imaging (AWL, KYT), Department of Pathology (SD, LCA), Department of Clinical Neurological Sciences (JAF, AFC), Department of Ophthalmology (JAF), Western University, London, Ontario, Canada.

Received October 11, 2013. Final Revisions Submitted January 22, 2014. Correspondence to: Amy W. Lin, Department of Medical Imaging, Western University, 800 Commissioners Road East, London, Ontario, N6A 5W9, Canada. Email: amy.lin@londonhospitals.ca. 


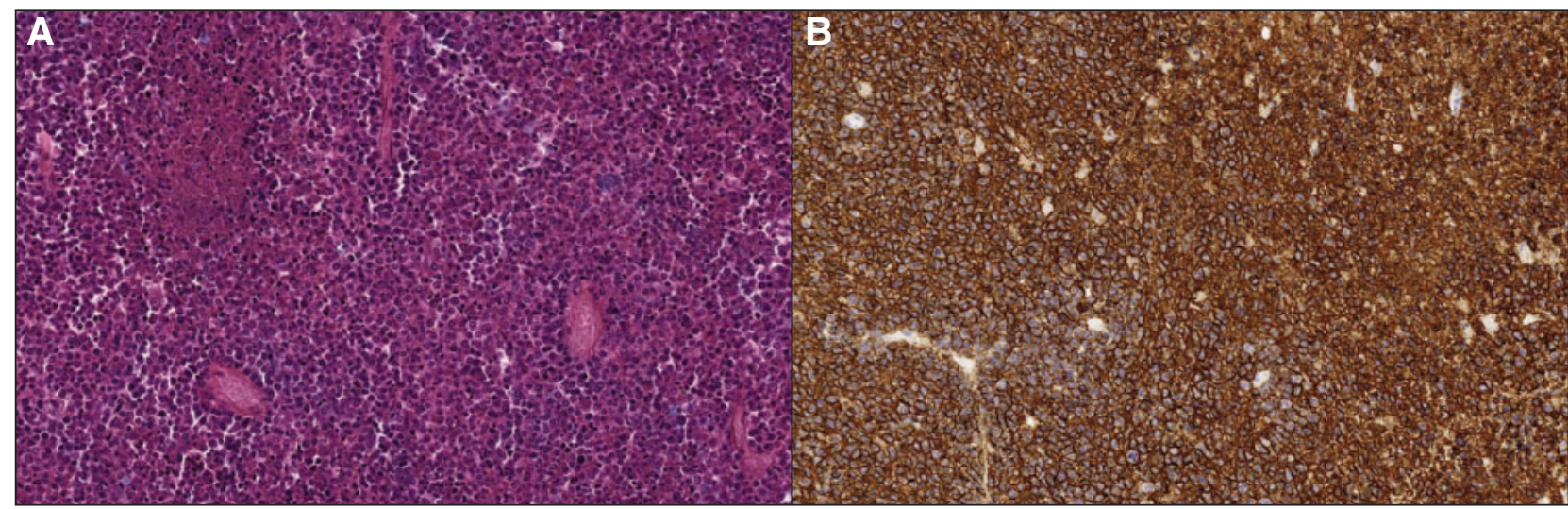

Figure 2: Representative histopathology image demonstrating a lymphoproliferative neoplasm (a) in which the neoplastic cells are diffusely immunoreactive for the B-cell immunomarker, CD20 (b).

oligoclonal bands, and no evidence for malignancy on cerebrospinal fluid (CSF) cytological assessments. Four CSF flow cytometry studies were done but these were all inconclusive due to inadequate numbers of B-lymphocytes. Serum and CSF angiotensin converting enzyme levels were normal. Infectious work-up was negative. Neuro-ophthalmic examination revealed no intraocular infiltration or granulomatous inflammation. Computed tomography (CT)-guided stereotactic needle biopsy of lesions in the left occipital white matter was performed with intraoperative frozen sections confirming biopsy of lesional tissue. Pathological analysis of samples showed patchy areas of predominantly perivascular mixed polyclonal lymphocytic infiltrate without evidence for lymphoma, glioma, granulomas, infection, or vasculitis. Computed tomography of the thorax, abdomen and pelvis showed several small non-specific pulmonary nodules without specific evidence for lymphoma or other malignancy.

Because a definitive alternative diagnosis could not be established, and because the radiological findings were suggestive, a working diagnosis of CLIPPERS was made, and the patient was treated with a five day course of $1 \mathrm{~g}$ IV methylprednisolone followed by $60 \mathrm{mg}$ of oral prednisone daily for one month and then azathioprine was added. He remained on both medications until his readmission to hospital. Within one to two days of receiving methylprednisolone, the patient's symptoms significantly improved and he was subsequently discharged from the hospital, with arrangements made for monthly outpatient follow-up. His symptoms continued to improve over the next three months on immunosuppressive treatment.

Unfortunately, at his four month follow-up, his symptoms started to return despite ongoing high-dose oral steroids, and the patient was readmitted to the inpatient neurology service with a subacute functional decline with right sided weakness, aphasia, and new urinary incontinence. Repeat MRI head (Figure 1b) showed a new focus of enhancement in the left thalamus and posterior limb of the internal capsule, much more in keeping with primary CNS lymphoma than CLIPPERS. His CSF flow cytometry and cytological studies were repeated and results were unchanged. The patient declined further investigations and elected for palliative care at this point. He continued to decline and died two weeks later. An autopsy was performed, which demonstrated large B-cell CNS lymphoma predominantly comprised of CD 20-positive neoplastic B-cells with scattered CD 3-positive reactive T-cells (Figure 2) in the pons, left putamen, left thalamus, and left posterior parietal lobe. Multifocal perivascular mixed lymphocytic infiltrates were seen distant from the areas of lymphoma, including at the site of the initial biopsy in the left occipital lobe.

\section{DISCUSSION}

A similar case has been previously reported of a patient with initial clinical, radiological, and pathological features compatible with CLIPPERS followed by the emergence of large B-cell lymphoma ${ }^{4}$. These two cases illustrate the challenges in diagnosing CLIPPERS, even when clinical and imaging features are suggestive of the disease and extensive workup shows no evidence for another process. It should be emphasized that the findings of polyclonal lymphocytic infiltrate on biopsy described in previous cases of CLIPPERS are non-specific and can be seen in multiple entities including the "sentinel" lesions of PCNSL", encephalitis, vasculitis and paraneoplastic syndromes. These pathological findings should therefore be correlated carefully with clinical findings and the results of other investigations, and close clinical follow-up of such patients is imperative.

\section{REFERENCES}

1. Pittock SJ, Debruyne J, Krecke KN, et al. Chronic lymphocytic inflammation with pontine perivascular enhancement responsive to steroids (CLIPPERS). Brain. 2010 Sep;133(9):2626-34.

2. Taieb G, Duflos C, Renard D, et al. Long-term outcomes of CLIPPERS (chronic lymphocytic inflammation with pontine perivascular enhancement responsive to steroids) in a consecutive series of 12 patients. Arch Neurol. 2012 Jul;69(7): 847-55

3. Simon NG, Parratt JD, Barnett MH, et al. Expanding the clinical, radiological and neuropathological phenotype of chronic lymphocytic inflammation with pontine perivascular enhancement responsive to steroids (CLIPPERS). J Neurol Neurosurg Psychiatry. 2012 Jan;83(1):15-22.

4. Limousin N, Praline J, Motica O, et al. Brain biopsy is required in steroid-resistant patients with chronic lymphocytic inflammation with pontine perivascular enhancement responsive to steroids (CLIPPERS). J Neurooncol. 2012 Mar;107(1):223-4.

5. Alderson L, Fetell MR, Sisti M, et al. Sentinel lesions of primary CNS lymphoma. J Neurol Neurosurg Psychiatry. 1996 Jan;60 (1):102-5. 